\title{
Diagnóstico de áreas agrícolas sob diferentes usos e manejos com o auxílio de ferramentas multivariadas
}

O presente estudo propõe a utilização de métodos quimiométricos como instrumento de determinação dos parâmetros de fertilidade, nutrição, micronutriente e física do solo mais relevantes em áreas agrícolas. A área de estudo está localizada no nordeste do estado do Pará, Brasil, são avaliadas tuas áreas, a primeira com Pimenta-do-Reino (Piper nigrum L.) e Açaí (Euterpe oleoracea), foram selecionadas uma gleba homogênea para realizar a coleta do solo, amostras deformadas e indeformadas, avaliando propriedades químicas, físicas e hídricas de ambos os solos. Os resultados foram submetidos a análise descritiva, teste $\mathrm{F}$ nos dados e posteriormente a Análise de Componentes Principais (ACP) e a Análise de Agrupamento Hierárquico (AAH), onde foi possível detectar parâmetros característicos para área e com isso auxiliar na tomada de decisão do manejo.

Palavras-chave: Análise de componentes principais; Agrupamento Hierárquico; Pimenta do reino; Açaí; Teste F.

\section{Diagnosis of agricultural areas under different uses and management using multivariate tools}

The present study proposes the use of chemometric methods as an instrument for determining the most relevant parameters of fertility, nutrition, micronutrients and soil physics in agricultural areas. The study area is located in the northeast of the state of Pará, Brazil, your areas are evaluated, the first with Black Pepper (Piper nigrum L.) and Açaí (Euterpe oleoracea), a homogeneous plot was selected to carry out the collection of the soil, deformed and deformed samples, evaluating chemical, physical and water properties of both soils. The results were submitted to descriptive analysis, $\mathrm{F}$ test on the data and later to Principal Component Analysis (PCA) and Hierarchical Cluster Analysis (HCA), where it was possible to detect characteristic parameters for the area and thereby assist in management decision making.

Keywords: Principal Component Analysis; Hierarchical Grouping; Black pepper; Açaí; Test F.

Topic: Ciências do Solo

Reviewed anonymously in the process of blind peer.
Received: 02/10/2020

Approved: $20 / 11 / 2020$
Mateus Higo Daves Alves (iD)

Universidade Federal Rural da Amazônia, Brasil http://lattes.cnpq.br/4912689548545873 http://orcid.org/0000-0001-9369-1338 mateushigo.alves@gmail.com

Pedro Moreira de Sousa Junior (iD) Universidade Federal Rural da Amazônia, Brasil http://lattes.cnpq.br/3194428833833524 http://orcid.org/0000-0002-6964-562X pedro.junior@ufra.edu.br

Joaquim Alves de Lima Junior (iD) Universidade Federal Rural da Amazônia, Brasil http://lattes.cnpq.br/0293681239695977 http://orcid.org/0000-0001-9003-7998 joaquim.junior@ufra.edu.br

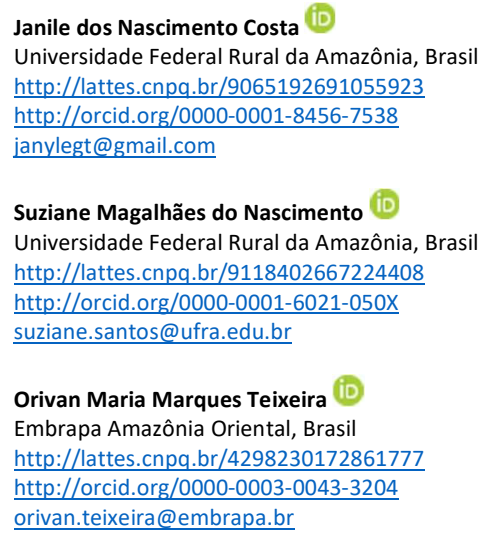

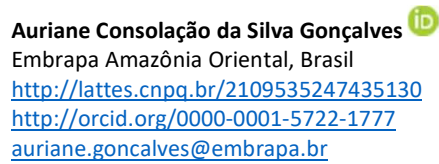

Referencing this:

ALVES, M. H. D.; SOUSA JUNIOR, P. M.; LIMA JUNIOR, J. A.; COSTA, J. N.; NASCIMENTO, S. M.; TEIXEIRA, O. M. M.; GONÇALVES, A. C. S. Diagnóstico de áreas agrícolas sob diferentes usos e manejos com o auxílio de ferramentas multivariadas. Revista Ibero Americana de Ciências Ambientais, v.11, n.6, p.13-22, 2020. DOI: http://doi.org/10.6008/CBPC2179-6858.2020.006.0002 


\section{INTRODUÇÃO}

Conhecer a saúde do solo é essencial para futuras ações de prevenção e melhoras do mesmo, de modo a proporcionar alimentos seguros respeitando sua capacidade (NEHRANI et al., 2020), e reconhecendo a influência nas condições físicas e químicas na agricultura sustentável (PANTOJA et al., 2019). Levando em consideração que a má gestão do solo pode levar a uma degradação irreversível (GOMES et al., 2019).

Na região amazônica a substituição das áreas de floresta por áreas agricultáveis continua sendo um das principais atividades do homem que causa bastante impacto sobre o solo quando ocorre de forma desordenada e sem os cuidados necessários (PANTOJA et al., 2019). Bem como os solos dessa região são de baixa fertilidade e reserva de nutrientes (CAMPOS et al., 2012) sendo dependentes da ciclagem dos nutrientes as áreas de mata (MOLINE et al., 2015). Desse modo as mudanças antrópicas no solo para a agricultura devem ser analisadas levando em consideração o manejo que é adotado, espécie cultivada, tempo que está sendo usado, e características típicas do solo, que podem atuar de maneira maléfica e benéfica (RAMOS et al., 2019).

Devido à complexidade de se trabalhar de se trabalhar com uma gama de dados e realizar a escolha do indicador nessa situação, adota-se métodos multivariados. A triagem realizada por este método seleciona um menor número de variáveis que são capazes de explicar significativamente a variância total do conjunto de dados, dessa maneira se reduz o número de variáveis que serram interpretadas e/ou selecionadas sem perder a qualidade dos dados (BRUNS et al., 1985). Nesse aspecto, o presente estudo propõe a utilização de métodos quimiométricos como instrumento de determinação dos parâmetros de fertilidade, nutrição, micronutrientes e física do solo mais relevantes em áreas agrícolas.

\section{MATERIAIS E MÉTODOS}

\section{Caracterização do local de estudo}

A fazenda Nova Esperança, está localizada na região nordeste do Pará, com coordenadas $1^{\circ} 10^{\prime} 11.53^{\prime \prime} \mathrm{S}$ e $47^{\circ} 59^{\prime} 17.98^{\prime \prime} \mathrm{O}$, altitude de $39 \mathrm{~m}$, haja vista que tem áreas voltadas para o cultivo de Pimenta do reino (Piper nigrum L.) e Açaí (Euterpe oleracea), ambas as áreas a serem estudadas.

\section{Amostragem do solo}

A amostragem de solo foi realizada em outubro de 2019. Em cada área agrícola (Pimenta do reino e Açaí) onde foi delimitada uma gleba representativa, composta de cinco unidades amostrais equidistantes de $10 \mathrm{~m}$, totalizando $100 \mathrm{~m}^{2}$, na profundidade de $0-0,20 \mathrm{~m}$, após remoção da camada de serapilheira foram realizadas as coletas de solo retirando amostras deformadas e indeformadas seguindo o proposto por Rossi et al. (2013).

\section{Análises Laboratoriais}

Os parâmetros químicos avaliados foram: $\mathrm{pH}$ em água $(1: 2,5)$, as bases trocáveis da solução do solo: 
Cálcio $\left(\mathrm{Ca}^{2+}\right)$, Magnésio $\left(\mathrm{Mg}^{2+}\right)$ e Potássio $\left(\mathrm{K}^{+}\right)$extraídos com $\mathrm{KCl} 1,0 \mathrm{~mol} \mathrm{~L}^{-1}$ por titulação, alumínio trocável $\left(\mathrm{Al}^{3+}\right)$ por volumetria e Fósforo (P) disponível (Mehlich-1), matéria orgânica do solo (Walkley Black), Nitrogênio Amoniacal $\left(\mathrm{NH}^{3}\right)$, micronutrientes: cobre $(\mathrm{Cu})$, Ferro $(\mathrm{Fe})$, Zinco $(\mathrm{Zn})$, Manganês $(\mathrm{Mn})$ extração com DTPA, posteriormente calculado a soma de bases (SB), capacidade de troca catiônica total (CTC) e saturação por alumínio (m\%) e por bases (V\%).

Os parâmetros físicos foram: porosidade total (PT), densidade do solo (Ds), frações texturais (argila, silte e areia), argila dispersa em água (ADA), teor de água disponível (AD), retenção de água na capacidade de campo $(0,1 \mathrm{~atm})$ e no ponto de murcha permanente (15 atm). Ambas analises desenvolvidas no laboratório da Embrapa - Amazônia Oriental.

\section{Analises estatísticas}

No tratamento dos dados foi utilizado o software, versão free, Minitab ${ }^{\circledR}$ Statistical versão 16 , foram utilizados análise descritiva, teste F nos dados e posteriormente a Análise de Componentes Principais (ACP) e a Análise de Agrupamento Hierárquico (AAH).

\section{RESULTADOS E DISCUSSÃO}

\section{Seleção de variáveis aplicando técnicas uni e multivariadas}

\section{Análise descritiva}

O resumo estatístico (tabela 1) proporcionado por esta técnica permitiu identificar-o parâmetro matéria orgânica $(\mathrm{MO})$ apresentou concentração com valor baixo $(<15 \mathrm{~g} / \mathrm{Kg})$ nas duas áreas pesquisadas, conforme esperado por Prezotti et al. (2013). Essa condição, também, foi observada em outros estudos em áreas agricultáveis na Amazônia (MOREIRA et al., 2004).

Sobre o grupo de parâmetros que compõem a subclasse fertilidade do solo, o estudo apresentou $\theta$ as seguintes variações pH $(5,0 \sim 5,3)$, Alumínio $(0,6 \sim 0,1)$, Acidez potencial $(4,3 \sim 3,3)$, Capacidade de troca catiônica $(5,3 \sim 5,8)$. Essas condições sugerem que os solos das áreas com plantio de Açaí e Pimenta do reino apresentam toxidez alta por conta do teor de Alumínio presente, em decorrência do pH baixo. Esta condição resulta em um $\mathrm{H}+\mathrm{Al}$ alto e CTC baixa. Nóia et al. (2014) afirmam que esse aspecto é comum em ambientes naturais e em áreas agrícolas com ausência de práticas conservacionistas.

No que diz respeito ao Fósforo disponível os teores identificados foram de 5,30 mg/dm na área de açaí e $33,13 \mathrm{mg} / \mathrm{dm}^{3}$ na área de pimenta do reino. Esses valores considerados são baixos a médio, segundo o estudo proposto por Brasil et al. (2007). A explicação, segundo Raij (1991), deve-se por conta da presença de alumínio e ferro no solo que por sua vez formam fosfatados de ferro e alumínio, inativando sua absorção pela planta, causando de baixa a média disponibilidade desse mineral no solo.

Os teores de ferro encontrados foram de 136,03 mg/Kg para área de açaí e 103,77 mg/Kg para área de pimenta do reino. Esses valores encontram-se acima dos níveis adequados propostos por Prezotti et al. (2013), e Brasil et al. (2007). Essa condição está relacionada a mineralogia do solo em regiões de clima tropical 
(RAIJ, 1991).

Tabela 1: Estatística descritiva dos parâmetros da subclasse fertilidade e nutrição do solo.

\begin{tabular}{|c|c|c|c|c|c|c|c|c|}
\hline \multirow{2}{*}{ Variáveis } & \multicolumn{3}{|c|}{ Cultivo de Açaí } & \multicolumn{3}{|c|}{$\begin{array}{l}\text { Cultivo de } \\
\text { Pimenta-do-Reino }\end{array}$} & \multirow[t]{2}{*}{$\begin{array}{l}\text { Brasil et al. } \\
(2007)\end{array}$} & \multirow[t]{2}{*}{$\begin{array}{l}\text { Prezotti et al. } \\
(2013)\end{array}$} \\
\hline & Média & $\begin{array}{l}\text { Erro } \\
\text { Padrão }\end{array}$ & $\begin{array}{l}\text { Desvio } \\
\text { Padrão }\end{array}$ & Média & $\begin{array}{l}\text { Erro } \\
\text { Padrão }\end{array}$ & $\begin{array}{l}\text { Desvio } \\
\text { Padrão }\end{array}$ & & \\
\hline $\mathrm{MO}(\mathrm{g} / \mathrm{Kg})$ & 11,420 & 0,071 & 0,175 & 13,375 & 0,075 & 0,184 & & $>3,0$ \\
\hline$P\left(\mathrm{mg} / \mathrm{dm}^{3}\right)$ & 5,300 & 0,071 & 0,175 & 33,130 & 0,071 & 0,175 & $11-30$ & $20-30$ \\
\hline $\mathrm{K}\left(\mathrm{mg} / \mathrm{dm}^{3}\right)$ & 12,230 & 0,50 & 1,225 & 11,628 & 0,564 & 1,382 & $46-90$ & $60-150$ \\
\hline $\mathrm{Al}\left(\mathrm{cmol}_{\mathrm{c}} / \mathrm{dm}^{3}\right)$ & 0,600 & 0,036 & 0,089 & 0,150 & 0,022 & 0,054 & $<0,2$ & $<0,3$ \\
\hline $\mathrm{Ca}+\mathrm{Mg}\left(\mathrm{cmol}_{\mathrm{c}} / \mathrm{dm}^{3}\right)$ & 0,950 & 0,076 & 0,187 & 2,483 & 0,047 & 0,116 & $2,1-6,0$ & \\
\hline $\mathrm{pH}$ & 5,001 & 0,064 & 0,157 & 5,395 & 0,028 & 0,069 & & $5,0-5,9$ \\
\hline $\mathrm{H}+\mathrm{Al}\left(\mathrm{cmol}_{\mathrm{c}} / \mathrm{dm}^{3}\right)$ & 4,378 & 0,020 & 0,050 & 3,333 & 0,008 & 0,021 & & $2,5-5,0$ \\
\hline $\begin{array}{ll}\text { CTC } & \text { total } \\
\left(\mathrm{cmol}_{\mathrm{c}} / \mathrm{dm}^{3}\right) & \\
\end{array}$ & 5,359 & 0,065 & 0,159 & 5,846 & 0,052 & 0,128 & & $4,5-10$ \\
\hline
\end{tabular}

Quanto ao elemento Zinco as concentrações determinadas de $2,7 \mathrm{mg} / \mathrm{Kg}$ e 7,3 mg/Kg nas áreas de açaí e pimenta do reino respectivamente (Tabela 2). Em relação ao Cobre os valores determinados foram de $0,8 \mathrm{mg} / \mathrm{Kg}$ na área de açaí e 3,3 mg/Kg na área de pimenta do reino, no que diz respeito a Manganês os teores encontrados foram de $6,7 \mathrm{mg} / \mathrm{Kg}$ na área de açaí e $11,53 \mathrm{mg} / \mathrm{Kg}$ na área de pimenta do reino. Os valores encontrados relacionados aos micronutrientes podem estar relacionados a matéria orgânica que foi encontrado, devido a retenção e disponibilidade dos mesmos através da MO e a decomposição da mesma pode libera esses micronutrientes (SILVEIRA et al., 2002).

Tabela 2: Estatística descritiva da subclasse micronutrientes.

\begin{tabular}{|c|c|c|c|c|c|c|c|c|}
\hline & \multicolumn{3}{|c|}{ Cultivo de Açaí } & \multicolumn{3}{|c|}{$\begin{array}{l}\text { Cultivo de } \\
\text { Pimenta-do-Reino }\end{array}$} & \multirow{2}{*}{ Brasil et al. (2007) } & \multirow{2}{*}{ Prezotti et al. (2013) } \\
\hline & Média & $\begin{array}{l}\text { Erro } \\
\text { Padrão }\end{array}$ & $\begin{array}{l}\text { Desvio } \\
\text { Padrão }\end{array}$ & Média & $\begin{array}{l}\text { Erro } \\
\text { Padrão }\end{array}$ & $\begin{array}{l}\text { Desvio } \\
\text { Padrão }\end{array}$ & & \\
\hline $\mathrm{Fe}(\mathrm{mg} / \mathrm{Kg})$ & 136,03 & 3,98 & 9,76 & 103,77 & 8,91 & 21,82 & $>45$ & $>46$ \\
\hline $\mathrm{Zn}(\mathrm{mg} / \mathrm{Kg})$ & 2,761 & 0,071 & 0,175 & 7,328 & 0,111 & 0,273 & $>2,2$ & $>2,2$ \\
\hline $\mathrm{Cu}(\mathrm{mg} / \mathrm{Kg})$ & 0,822 & 0,063 & 0,155 & 3,388 & 0,093 & 0,229 & $0,70-1,80$ & $0,70-1,80$ \\
\hline $\begin{array}{l}\mathrm{Mn} \\
(\mathrm{mg} / \mathrm{Kg})\end{array}$ & 6,767 & 0,141 & 0,344 & 11,533 & 0,098 & 0,242 & $5-12$ & $5-12$ \\
\hline
\end{tabular}

Na tabela 3, ao qual estão dispostos os resultados da subclasse física do solo, os valores de porosidade total determinados foram de $0,45 \mathrm{~m}^{-3} / \mathrm{m}^{-3}$ no cultivo de açaí e $0,47 \mathrm{~m}^{3} \mathrm{~m}^{-3}$ no cultivo de pimentado-reino esses valores são considerados baixos quando comparado ao valor ideal proposto por Kiehl (1979). Esse resultado deve-se a fração areia o solo apresenta e o tipo de manejo adotado, que condensa e aumenta a porosidade (BRASIL NETO et al., 2018; OLIVEIRA JUNIOR et al., 1997).

No que diz respeito a densidade do solo os valores identificados foram de $1,37 \mathrm{~kg} \cdot \mathrm{dm}^{-3}$ no cultivo de Açaí e 1,43 kg.dm ${ }^{-3}$ para Cultivo da pimenta do reino, esses valores são considerados baixos $\left(1,65 \mathrm{~kg} \cdot \mathrm{dm}^{-3}\right)$ do limite critico de desenvolvimento das culturas, conforme proposto por (REICHERT et al., 2003), levando em consideração solo com valores de argila menor que $200 \mathrm{~g}$. É valido ressaltar que a diferença de valores das áreas está relacionada com a exposição do solo após a roçagem na entrelinha dos plantios que causa pisoteio que provoca o aumento da compactação.

Sobre a Agua Disponível (AD), os valores encontrados foram de 0,14 no cultivo de açaí e de 0,08 para 
o Cultivo de Pimenta-do-Reino, esses valores estão abaixo do adequado $(<0,15)$ em ambas as áreas, conforme proposto por Cockrooft et al. (1997). Com base na quantidade de areia, silte e argila, ambas as áreas foram classificadas segundo a EMBRAPA (2018) como Latossolo amarelo distrófico franco arenoso.

Tabela 3: Resultados dos parâmetros referente a subclasse física e hídrica do solo.

\begin{tabular}{|c|c|c|c|c|c|c|c|c|c|}
\hline \multirow{2}{*}{ Variáveis } & \multicolumn{3}{|c|}{ Cultivo de Açaí } & \multicolumn{3}{|c|}{$\begin{array}{l}\text { Cultivo de } \\
\text { Pimenta-do-Reino }\end{array}$} & \multirow[t]{2}{*}{$\begin{array}{l}\text { Reichert et al. } \\
(2003)\end{array}$} & \multirow[t]{2}{*}{$\begin{array}{l}\text { Kiehl } \\
\text { (1979) }\end{array}$} & \multirow[t]{2}{*}{$\begin{array}{l}\text { Cockrooft et al. } \\
(1997)\end{array}$} \\
\hline & Média & $\begin{array}{l}\text { Erro } \\
\text { Padrão }\end{array}$ & $\begin{array}{l}\text { Desvio } \\
\text { Padrão }\end{array}$ & Média & $\begin{array}{l}\text { Erro } \\
\text { Padrão }\end{array}$ & $\begin{array}{l}\text { Desvio } \\
\text { Padrão }\end{array}$ & & & \\
\hline $\begin{array}{l}\text { Ds }(\mathrm{kg} \mathrm{dm}- \\
\text { 3) }\end{array}$ & 1,37 & 0,041 & 0,101 & 1,43 & 0,026 & 0,063 & $1,6-1,8$ & & \\
\hline $\begin{array}{l}\text { PT }\left(\mathrm{m}^{-3} / \mathrm{m}^{-}\right. \\
\text {3) }\end{array}$ & 0,45 & 0,527 & 1,291 & 0,47 & 1,67 & 4,09 & & 0,50 & \\
\hline$A D$ & 0,14 & 0,013 & 0,034 & 0,08 & 0,008 & 0,020 & & & $0,15-0,25$ \\
\hline Areia & 824,33 & 0,476 & 1,12 & 817,33 & 1,61 & 3,93 & & & \\
\hline Silte & 55,667 & 0,394 & 1,21 & 64,33 & 0,49 & 1,21 & & & \\
\hline Argila & 120,00 & 0,000 & 0,000 & 120,00 & 0,000 & 0,000 & & & \\
\hline$-30 \mathrm{kPa}$ & 0,193 & 0,0151 & 0,036 & 0,143 & 0,007 & 0,017 & & & \\
\hline$-1500 \mathrm{kPa}$ & 0,052 & 0,002 & 0,005 & 0,059 & 0,002 & 0,006 & & & \\
\hline
\end{tabular}

\section{Teste F}

De acordo com Borges et al. (2003) o teste F analisa a hipótese de similaridades entre as médias dos grupos, se são ou não iguais estatisticamente, no entanto não permite saber, quando a diferença, quais se diferenciam e/ou agrupam quando se trabalha com mais de dois grupos, que não é o caso do presente estudo. Dentro da comparação entre as áreas foi evidenciada existência de diferença significativa das variáveis (tabela 4): Matéria Orgânica, Fósforo, Alumínio, Cálcio e Magnésio, Acidez potencial, Capacidade de Troca Catiônica, Ferro, Zinco, Cobre, Manganês, Areia, Silte e água disponível.

Tabela 4: Resultados da aplicação do teste F.

\begin{tabular}{lll}
\hline Variáveis & $\mathrm{F}$ & Significância \\
\hline Matéria orgânica $(\mathrm{g} / \mathrm{Kg})$ & 354,603 & $<0,05$ \\
Fósforo $\left(\mathrm{mg} / \mathrm{dm}^{3}\right)$ & 75537,279 & $<0,05$ \\
Potássio $\left(\mathrm{cmol}_{\mathrm{c}} / \mathrm{dm}^{3}\right)$ & 0,636 & 0,444 \\
Alumínio & 110,455 & $<0,05$ \\
Cálcio e Magnésio & 289,863 & $<0,05$ \\
$\mathrm{pH}$ & 1,099 & 0,319 \\
Acidez potencial & 2152,952 & $<0,05$ \\
CTC total & 33,772 & $<0,05$ \\
Ferro & 10,931 & $<0,05$ \\
Zinco & 1194,489 & $<0,05$ \\
Cobre & 514,854 & $<0,05$ \\
Manganês & 768,759 & $<0,05$ \\
Areia & 17,362 & $<0,05$ \\
Silte & 153,636 & $<0,05$ \\
-30 kPa & 2,927 & 0,140 \\
-1500 kPa & 3,983 & 0,074 \\
Densidade do solo & 1,363 & 0,270 \\
Porosidade total & 2,409 & 0,152 \\
Água disponível & 12,688 & $<0,05$ \\
\hline
\end{tabular}

\section{Análise por métodos multivariados}

O uso de estatística uni variada proporciona dúvidas em relação a avaliação dos fatores do solo, pois é limitada na análise visto que aborda o comportamento isolado, desconsiderando as interações com as 
demais variáveis de forma simultânea (SILVA et al., 2010).

Análise multivariada é uma ferramenta que normalmente auxilia nesta lacuna por agrupar amostras e variáveis de acordo com a similaridade e avaliar as interações não mais de forma isolada como na uni variada (BENITES et al., 2010). Segundo James et al. (1990) as técnicas multivariadas são aplicáveis quando se tem um conjunto com grande número de variáveis em um único estudo, que são submetidas a estatísticas de inferência, exploratória e descritiva para analisar as variáveis.

\section{Análise de Componentes Principais (ACP)}

Esta ferramenta tornou-se valiosa para o estudo após analisar que autores como Oliveira et al. (2017), que trabalharam em áreas semelhantes, apresentarem ótimos resultados interpretativos fundamentados nesse teste. O potencial dessa ferramenta foi mostrado no estudo de Armenise et al. (2013) em que utilizou variáveis físicas, químicas e biológicas na avaliação de qualidade do solo.

O tratamento estatístico aplicado foi no formato bidimensional que leva em consideração somente a primeira e segunda componente, tomando como base para seleção de variáveis. Partindo de um conjunto com dezenove variáveis, foi possível reduzir para doze variáveis, contendo a percentagem de $94 \%$ de variância acumulada e apenas $6 \%$ de informação não explicada (tabela 5). Não há padrão sobre o percentual de variância acumulada, embora autores como Hair et al. (2005) recomendem variância acumulada seja maior a 70\% com o mínimo de componentes possível.

A primeira componente (CP1) teve como variáveis que mais contribuíram para a sua variância, levando em consideração as cargas fatoriais, o Fósforo, Manganês, Alumínio, Zinco, Cálcio + Magnésio, Matéria Orgânica, Acidez potencial e a areia explicando $88,10 \%$ de variância. No segundo componente as variáveis foram: Ferro, água disponível, Silte e o Cobre com 6\% de explicação.

Tabela 5: Pesos das duas primeiras componentes principais selecionadas.

\begin{tabular}{lll}
\hline COMPONENTE & CP1 & CP2 \\
\hline Proporção (\%) & 88,1 & 6,00 \\
Acumulado (\%) & 88,1 & 94,1 \\
VARIÁVEIS & & 0,009 \\
\hline Matéria orgânica $(\mathrm{g} / \mathrm{Kg})$ & $0,301^{\mathrm{a}}$ & 0,019 \\
Potássio $\left(\mathrm{cmol}_{\mathrm{c}} / \mathrm{dm}^{3}\right)$ & $0,306^{\mathrm{a}}$ & 0,023 \\
Alumínio $\left(\mathrm{cmol}_{\mathrm{c}} / \mathrm{dm}^{3}\right)$ & $-0,299^{\mathrm{a}}$ & 0,012 \\
Cálcio e Magnésio $\left(\mathrm{cmol}_{\mathrm{c}} / \mathrm{dm}^{3}\right)$ & $0,303^{\mathrm{a}}$ & $-0,003$ \\
Acidez potencial $\left(\mathrm{cmol}_{\mathrm{c}} / \mathrm{dm}^{3}\right)$ & $-0,306^{\mathrm{a}}$ & $0,748^{\mathrm{b}}$ \\
Ferro $(\mathrm{mg} / \mathrm{Kg})$ & $-0,226$ & $-0,013$ \\
Zinco $(\mathrm{mg} / \mathrm{Kg})$ & $0,306^{\mathrm{a}}$ & $0,114^{\mathrm{b}}$ \\
Cobre $(\mathrm{mg} / \mathrm{Kg})$ & 0,302 & 0,082 \\
Manganês $(\mathrm{mg} / \mathrm{Kg})$ & $0,304^{\mathrm{a}}$ & $-0,600^{\mathrm{b}}$ \\
Areia & $-0,249$ & 0,051 \\
Silte & $0,301^{\mathrm{a}}$ & $0,237^{\mathrm{b}}$ \\
Água disponível & $-0,245$ & 2 \\
\hline
\end{tabular}

a: Elementos que formam a Componente Principal 1 (CP1); b: Elementos que formam a Componente Principal 2 (CP2).

A figura 1 ilustra a aglomeração das variáveis e das amostras, no plano bidimensional (2D), evidenciando dois agrupamentos formados. O primeiro constituído pelas variáveis da subclasse fertilidade do solo representada pelos parâmetros Ferro, Água Disponível, Alumínio, Acidez Potencial e Areia, o segundo 
grupo constituído pela subclasse nutrição aos quais destaca-se Fósforo, Cálcio + Magnésio, Cobre, Manganês, Zinco, silte e Matéria orgânica. Com esses agrupamentos de variáveis foi possível identificar formação de agrupamentos correspondentes ao cultivo de Açaí e ao de Pimenta do Reino.

As variáveis da subclasse fertilidade do solo (acidez potencial e Alumínio) destacaram-se na área de açaí, fato este, que pode ter relação com o longo período sem calagem (CIOTTA et al., 2002). Todavia, uma das características dos solos amazônicos é apresentar a acidez devido à alta intemperização, provocadas pela lixiviação (MANTOVANELLI et al., 2016).

Da subclasse física e hídrica do solo (água disponível e areia) estão ligadas a questões hídricas, o que confirma as a baixas disponibilidade desta área. Solos arenosos são altamente permeáveis e com baixa capacidade de retenção de água, baixo teor de matéria orgânica, estão mais propensos a erosão, embora a adoção de práticas conservacionistas podem reduzir a susceptibilidade do mesmo (BRADY et al., 2013). A água no solo pode ser afetada pela textura, haja vista que adesão e coesão de partícula é afetada e consequentemente afeta a retenção de água, uma vez que a textura é intrínseca ao solo, não está sujeita mudança (CENTENO et al., 2017).

Em relação ao segundo aglomerado, o mesmo está atrelado a subclasse nutrição do solo, matéria orgânica, sendo ela oriunda de resíduos vegetais e animais (PEREIRA et al., 2010), a qual pode ter disponibilizado os elementos químicos que se destacaram (Fósforo, cálcio e magnésio). Em relação ao cobre, zinco e manganês, esses elementos são oriundos do material de origem do solo e são disponibilizados conforme o intemperismo (SHENG et al., 2012).

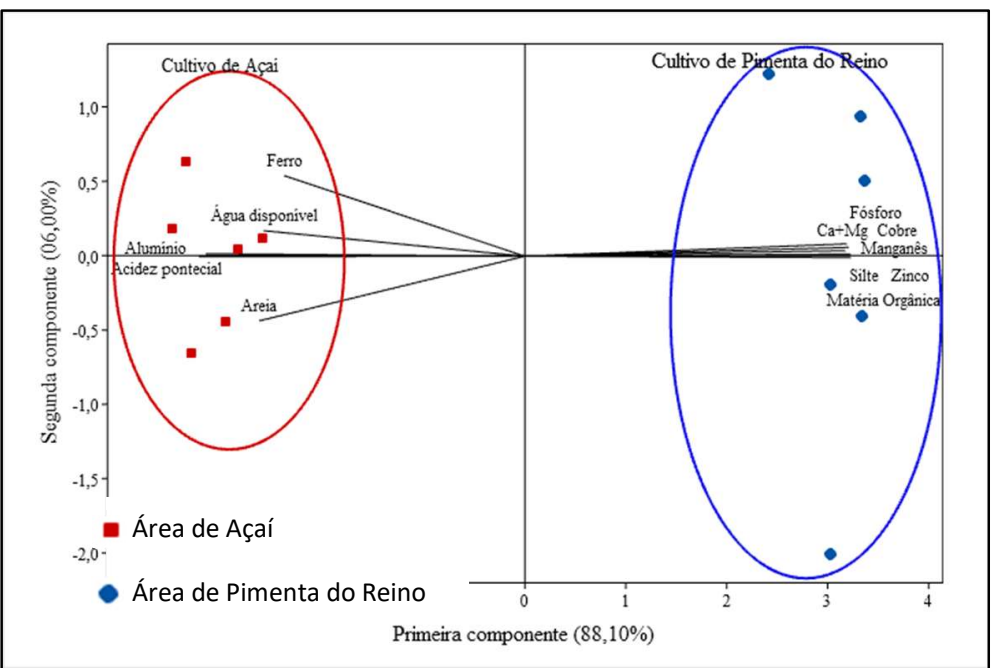

Figura 1: Variáveis (loadings) e amostras (scores) ilustra o agrupamento das variáveis entre as duas primeiras componentes.

\section{Análise de Agrupamento Hierárquico}

É uma técnica estatística usada para classificar as variáveis em grupo de forma que cada cluster apresente variáveis com similaridades e elementos em cluster diferentes apresentam diferenças, tendo como principal característica evidenciar em um plano 2D os agrupamentos e padrões naturais das variáveis levando em consideração a similaridade entres as mesmas (CORREIA et al., 2007).

Pantoja et al. (2019) reforça que a capacidade do solo, o uso e o manejo adotado, altera as 
propriedades químicas e físicas que proporciona a formação de grupos distintos através das similaridades entre as mesmas, que pode ser visto também através do dendrograma.

Com o intuito de identificar a semelhança entre as variáveis, utilizou-se essa Análise de Agrupamento Hierárquico (AAH) e obteve-se a formação dos agrupamentos (Figura 2) das variáveis que apresentaram diferença significativa pelo teste t e as que tem maior número de correlações.

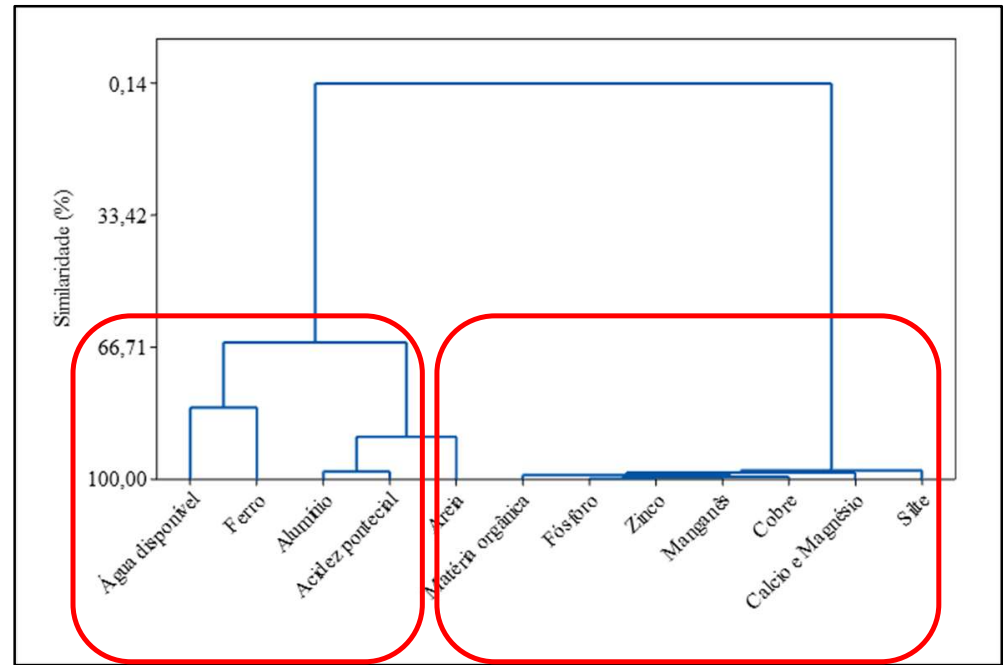

Figura 2: Dendrograma de agrupamento das variáveis selecionadas.

\section{CONCLUSÕES}

As áreas estão com parâmetros com valores abaixo da média recomendado pela literatura, assim como apresentam particularidades em relação a alguns parâmetros e diferenças em relação a outros, que por sua vez foram observados através de técnicas multivariadas, que proporcionou selecionar treze variáveis que mais contribuíram para a variabilidade dos dados, deste modo se conheceu as variáveis importantes estatisticamente para o estudo.

\section{REFERÊNCIAS}

ARMENISE, E.; REDMILE-GORDON, M. A.; STELLACCI, A. M.; CICCARESE, A.; RUBINO, P.. Developing a soil quality index to compare soil fitness for agricultural use under different managements in the Mediterranean environment. Soil and Tillage Research, v.130, p.91-98, 2013. DOI: http://doi.org/10.1016/j.still.2013.02.013

BENITES, V. M.; MOUTTA, R. O.; COUTINHO, H. L. C.; BALIEIRO, F. C.. Análise discriminante de solos sob diferentes usos em área de Mata Atlântica a partir de atributos da matéria orgânica. Revista Árvore, v.34, p.685-690, 2010. DOI: http://doi.org/10.1590/S0100-67622010000400013

BORGES, L. C.; FERREIRA, D. F.. Poder e taxas de erro tipo I dos testes Scott-Knott, Tukey e Student-Newman-Keuls sob distribuições normal e não normais dos resíduos. Revista de matemática e estatística, v.21, n.1, p.67-83, 2003.

BRADY, N. C.; WEIL, R. R.. Elementos da natureza e propriedades dos solos. 3 ed. Porto Alegre: Bookman, 2013.

BRASIL NETO, A. B.; SANTOS, C. R. C.; NORONHA, N. C.;
GAMA, M. A. P.; CARVALHO, E. J. M.; SILVA, A. R.; GUIMARAES, I. V. P.; SOUZA, P. Í. A.. Matéria orgânica e atributos físico-hídricos de um latossolo sob diferentes sistemas de manejo. Revista Agroecossistemas, v.10, n.2, p.147-164, 2018. DOI:

https://doi.org/10.18542/ragros.v10i2.5134

BRASIL, E. C.; CRAVO, M. S.. Interpretação dos Resultados de Análise de Solo. In: CRAVO, M. S; VIÉGAS, I. J. M.; BRASIL, E. C.. Recomendações de Adubação e Calagem para o Estado do Pará. Belém, 2007.

BRUNS, R. E.; FAIGLE, J. F. G.. Quimiometria. Química Nova, v.8, n.2, p.84-99, 1985.

CAMPOS, M. C. C.; SANTOS, L. A. C.; SILVA, D. M. P.; MANTOVANELLI, B. C.; SOARES, M. D. R.. Caracterização física e química de terras pretas arqueológicas e de solos não antropogênicos na região de Manicoré, Amazonas. Revista Agro@mbiente, v.6, n.2, p.102-109, 2012. DOI: http://dx.doi.org/10.18227/1982-8470ragro.v6i2.682 
CENTENO, L. N.; GUEVARA, M. D. F.; CECCONELLO, S. T.; SOUSA, R. O.; TIMM, L. C.. Textura do solo: conceitos e aplicações em solos arenosos. Revista Brasileira de Engenharia e Sustentabilidade, v.4, p.31-37, 2017. DOI: http://dx.doi.org/10.15210/rbes.v4i1.11576

CIOTTA, M. N.; BAYER, C.; ERNANI, P. R.; FONTOURA, S. M. V.; ALBUQUERQUE, J. A.; WOBETO, C.. Acidificação de um Latossolo sob plantio direto. Revista Brasileira de Ciência do Solo, v.26, p.1055-1064, 2002. DOI: http://dx.doi.org/10.1590/S0100-06832002000400023

COCKROOFT, B.; OLSSON, K. A.. Case study of soil quality in south-eastern Australia: management of structure for roots in duplex soils. In: GREGORICH, E. G.; CARTER, M. R.. Soil quality for crop production and ecosystem health developments in soil science. New York: Elsevier, 1997. p.339-350.

CORREIA, P. R. M.; FERREIRA, M. M. C.. Reconhecimento de padrões por métodos não supervisionados: explorando procedimentos quimiométricos para tratamento de dados analíticos. Química Nova, v.30, p.481-487, 2007. DOI: http://dx.doi.org/10.1590/S0100-40422007000200042

EMBRAPA. Empresa Brasileira de Pesquisa Agropecuária. Sistema brasileiro de classificação de solos. Brasília: EMBRAPA, 2018.

GOMES, R. P.; BERGAMIN, A. C.; SILVA, L. S.; CAMPOS, M. C. C.; CAZETTA, J. O.; COELHO, A. P.; SOUZA, E. D. D.. Changes in the physical properties of an Amazonian Inceptisol induced by tractor traffic. Chilean Journal of Agricultural Research, v.79, n.1, p.103-113, 2019. DOI: http://dx.doi.org/10.4067/S0718-58392019000100103

HAIR, J. R.; ANDERSON, R. E.; TATHAM, R. L.; BLACK, W. C.. Análise multivariada de dados. Porto Alegre: Bookman, 2005.

JAMES, F. C.; MCCULLOCH, C. E.. Multivariate Analysis in Ecology and Systematics: Panacea or Pandora's Box?. Annual Review of Ecology and Systematics, v.21, p.129-166, 1990. DOI:

http://dx.doi.org/10.1146/annurev.es.21.110190.001021

SHENG, J. J.; WANG, X. P.; GONG, P.; TIAN, L. D.; YAO, T. D. Heavy metals of the Tibetan top soils Level, source, spatial distribution, temporal variation and risk assessment. Environmental Science and Pollution Research, v.19, n.8, p.3362-3370, 2012. DOI: https://doi.org/10.1007/s11356$\underline{012-0857-5}$

KIEHL, E. J.. Manual de Edafologia; relações solo-planta. Ceres, 1979.

MANTOVANELLI, B. C.; CAMPOS, M. C. C.; ALHO, L. C.; FRANCISCON, U.; NASCIMENTO, M. F.; SANTOS, L. A. C.. Distribuição espacial dos componentes da acidez do solo em área de campo natural na região de Humaitá, Amazonas. Revista de Ciências Agroambientais, v.14, 2016.

MOLINE, E. F. V.; COUTINHO, E. L. M.. Atributos químicos de solos da Amazônia Ocidental após sucessão da mata nativa em áreas de cultivo. Revista de Ciências Agrárias: Amazonian Journal of Agricultural and Environmental Sciences, v.58, n.1, p.14-20, 2015. DOI: http://dx.doi.org/10.4322/rca.1683

MOREIRA, A.; MALAVOLTA, E.. Dinâmica da matéria orgânica e da biomassa microbiana em solo submetido a diferentes sistemas de manejo na Amazônia Ocidental. Pesquisa Agropecuária Brasileira, v.39, n.11, p.1103-1110, 2004. DOI: https://doi.org/10.1590/S0100-204X2004001100008

NEHRANI, S. H.; ASKARI, M. S.; SAADAT, S.; DELAVAR, M. A.; TAHERI, M.; HOLDEN, N. M.. Quantification of soil quality under semi-arid agriculture in the northwest of Iran. Ecological Indicators, v.108, p.105770, 2020. DOI: https://doi.org/10.1016/j.ecolind.2019.105770

NÓIA, N. R. C.; CRUZ, M. C. P.; DAVALO, M. J.; OLIVEIRA, M. C.; FUJITA, C. K. Produção de matéria seca de alfafa (Medicago sativa L.) em função de doses de calcário. Científica, v.42, p.310-315, 2014. DOI: http://dx.doi.org/10.15361/1984-5529.2014v42n3p310-315

OLIVEIRA JÚNIOR, R. C.; SILVA, J. M. L.; CAPECHE, C.; RODRIGUES, T.. Levantamento de reconhecimento de alta intensidade dos solos da folha Marapanim, Estado do Pará. Embrapa Amazônia Oriental, 1997.

OLIVEIRA, I. A.; MARQUES JÚNIOR, J.; CAMPOS, M. C. C.; AQUINO, R. E.; FREITAS, L.; FERRAUDO, A. S.. Multivariate technique for determination of soil pedoenvironmental indicators in Southern Amazonas. Acta Scientiarum. Agronomy, v.39, p.99, 2017. DOI: http://dx.doi.org/10.4025/actasciagron.v39i1.30763

PANTOJA, J. C. M.; CAMPOS, M. C. C.; LIMA, A. F. L. D.; CUNHA, J. M. D.; SIMÕES, E. L.; OLIVEIRA, I. A. D.; SILVA, L. S.. Multivariate analysis in the evaluation of soil attributes in areas under different uses in the region of Humaitá, AM. Revista Ambiente \& Água, v.14, n.5, 2019. DOI: https://doi.org/10.4136/ambi-agua.2342

PEREIRA, M. G.; LOSS, A.; BEUTLER, S. J.; TORRES, J. L. R.. Carbono, matéria orgânica leve e fósforo remanescente em diferentes sistemas de manejo do solo. Pesquisa agropecuaria brasileira, v.45, n.5, p.508-514, 2010. DOI: https://doi.org/10.1590/S0100-204X2010000500010

PREZOTTI, L. C.; MARTINS, A. G.. Guia de interpretação de análise de solo e foliar. Vitória: Incaper, 2013.

RAIJ, B. V.. Fertilidade do solo e adubação. Piracicaba: Associação Brasileira para Pesquisa do Potássio e do Fosfato, 1991.

RAMOS, A. M. R.; AMORIM, B. M. B.; FREIRE, C. T. M.; LIMA, D. L. F. A.. Atributos físicos do solo em sistema consorciado e monocultivo do maracujá (Passiflora edulis sims). Revista Brasileira de Engenharia de Biossistemas, v.13, n.1, p.80-87, 2019. DOI:

http://dx.doi.org/10.18011/bioeng2019v13n1p80-87

REICHERT, J. M.; REINERT, D. J.; BRAIDA, J. A.. Qualidade dos solos e sustentabilidade de sistemas agrícolas. Ci. Amb, v.27, p.29-48, 2003.

ROSSI, C. Q.; PEREIRA, M. G.; LOSS, A.; GAZOLLA, P. R.; PERIN, A.; ANJOS, L. H. C.. Changes in soil C and N distribution assessed by natural $\delta 13 \mathrm{C}$ and $\delta 15 \mathrm{~N}$ abundance in a chronosequence of sugarcane crops managed with pre- 
harvest burning in a Cerrado area of Goiás,

Brazil. Agriculture, Ecosystems \& Environment, v.170, p.36-

44, 2013. DOI: https://doi.org/10.1016/i.agee.2013.03.008

SILVA, M. O.; FREIRE, M. B. G. S.; MENDES, A. M. S.; FREIRE, F. J.; CAMPOS, M. C. C.; AMORIM, L. B.. Discriminação de diferentes classes de solos irrigados com águas salinas, na região de Mossoró (RN), com o uso de análise multivariada.
Ambiência, v.6, p.261-270, 2010.

SILVEIRA, P. M.; CUNHA, A. A.. Variabilidade de micronutrientes, matéria orgânica e argila de um Latossolo submetido a sistemas de preparo. Pesquisa Agropecuária Brasileira, v.37, n.9, p.1325-1332, 2002. DOI: http://dx.doi.org/10.1590/S0100-204X2002000900017

A CBPC - Companhia Brasileira de Produção Científica (CNPJ: 11.221.422/0001-03) detém os direitos materiais desta publicação. Os direitos referem-se à publicação do trabalho em qualquer parte do mundo, incluindo os direitos às renovações, expansões e disseminações da contribuição, bem como outros direitos subsidiários. Todos os trabalhos publicados eletronicamente poderão posteriormente ser publicados em coletâneas impressas sob coordenação da Sustenere Publishing, da Companhia Brasileira de Produção Científica e seus parceiros autorizados. Os (as) autores (as) preservam os direitos autorais, mas não têm permissão para a publicação da contribuição em outro meio, impresso ou digital, em português ou em tradução. 